\title{
The Effect of Sodium lodide on Stromal Loading, Distribution and Degradation of Riboflavin in a Rabbit Model of Transepithelial Corneal Crosslinking
}

\author{
Roy S Rubinfeld' \\ Glenwood G Gum² \\ Jonathan H Talamo (D) ${ }^{1,3}$ \\ Edward C Parsons (ID) \\ 'CXL Ophthalmics, LLC, Encinitas, CA, \\ USA; ${ }^{2}$ Absorption Systems California, \\ LLC, San Diego, CA, USA; ${ }^{3}$ Department \\ of Ophthalmology, Massachusetts Eye and \\ Ear, Harvard Medical School, Boston, \\ MA, USA
}

\begin{abstract}
Purpose: To evaluate effects of sodium iodide (NaI) on riboflavin concentration in corneal stroma before and during ultraviolet A (UVA) light exposure using a novel transepithelial corneal collagen crosslinking (CXL) procedure (EpiSmart CXL system, CXL Ophthalmics, Encinitas CA).

Methods: Riboflavin solutions with NaI (Ribostat, CXL Ophthalmics, Encinitas CA) and without $\mathrm{NaI}$ were used for CXL in rabbits using EpiSmart. A pilot study determined sufficient riboflavin loading time. Four rabbits were dosed and monitored. Riboflavin fluorescence intensity was assessed from masked slit-lamp photos. A 12 min loading time was selected. Sixteen additional rabbits received the two formulae in contralateral eyes for CXL. Riboflavin uptake was assessed at 0,10, 15, 20, 25, and 30 min of UVA exposure using a scale for riboflavin fluorescence previously validated against stromal concentration. Post sacrifice, corneal stromal samples were analyzed for concentrations of riboflavin and riboflavin 5'-phosphate.
\end{abstract}

Results: Eyes dosed with NaI riboflavin had higher riboflavin grades compared to eyes dosed with the NaI-free riboflavin formulation immediately after riboflavin loading and persisting throughout UVA exposure, with significantly higher $(P<0.01$ to $<0.05)$ riboflavin grades from 15 through 25 min of UVA exposure. Riboflavin grades decreased more slowly in eyes dosed with NaI riboflavin through 25 minutes of UVA exposure. Minor conjunctival irritation was noted with or without $\mathrm{NaI}$

Conclusion: The addition of $\mathrm{NaI}$ to riboflavin solution is associated with increased riboflavin concentration in corneal stroma throughout a clinically relevant time course of UVA exposure. This effect may be a combination of enhanced epithelial penetration and reduced riboflavin photodegradation and should enhance intrastromal crosslinking.

Keywords: corneal crosslinking, crosslinking, riboflavin, iodide, keratoconus, ectasia

\section{Introduction}

Corneal crosslinking (CXL) is an important treatment for ectatic corneal diseases, particularly keratoconus and postoperative keratectasia. ${ }^{1}$ The use of riboflavin, activated by ultraviolet A (UVA) exposure, induces crosslinking; the formation of covalent bonds between adjacent stromal proteins and proteoglycans. ${ }^{2}$ This provides biomechanical strengthening of treated corneas and increases their resistance to enzymatic digestion. First developed in the late $1990 \mathrm{~s},{ }^{3}$ CXL is now well established as an efficacious procedure. ${ }^{4-7}$ However, the standard CXL procedure
Correspondence: Roy S Rubinfeld

CXL Ophthalmics, LLC, 3I3I

Connecticut Avenue NW \#2809,

Washington DC, 20008, USA

$\mathrm{Tel}+1240305-1010$

Email rsrubinfeld@gmail.com 
entails surgical removal of the corneal epithelium to facilitate riboflavin delivery into the corneal stroma (epi-off CXL). Epithelial removal leads to pain and limited visual function that can persist for weeks and months, respectively, as the epithelial layer heals. ${ }^{8,9}$ Corneal haze affects over $90 \%$ of eyes after epi-off CXL, lasting up to 6 months ${ }^{10}$ or longer. ${ }^{11}$ Other risks include edema, infectious keratitis, corneal perforation, and corneal scarring. ${ }^{12}$ An effective CXL procedure not requiring epithelial removal (epi-on CXL) would be safer and less disruptive for the patient.

Riboflavin is a negatively charged, hydrophilic molecule with low corneal epithelial permeability. ${ }^{13}$ Topical application of riboflavin formulations onto intact epithelium typically do not produce sufficient intrastromal riboflavin concentrations for successful CXL. ${ }^{14,15}$ Several approaches to overcoming this limitation have been explored, including increased exposure time, ${ }^{14}$ permeation enhancers such as ethanol, ${ }^{16}$ preservatives, ${ }^{15,17}$ chelating agents, ${ }^{15,18,19}$ local anesthetics, ${ }^{20,21}$ emulsifiers, ${ }^{22}$ vitamin $\mathrm{E},{ }^{15,23,24}$ partial epithelial disruption, ${ }^{16,25-27}$ iontophoresis ${ }^{28}$ and ultrasound. $^{29}$ While these approaches do increase the absorption of riboflavin across the corneal epithelium, the resulting stromal riboflavin concentrations generally remain below those obtained after epithelial removal. ${ }^{15,16,21,28-30}$ Initially promising early clinical results (up to 12 months) with some techniques ${ }^{31-34}$ were followed by disappointing long-term clinical results at 2-3 years follow-up. , $^{19,35,36}$

Partial mechanical disruption of the epithelium is associated with complications similar to full epithelial removal. ${ }^{37}$ Moreover, epithelial disruption or postoperative epithelial sloughing with some permeation enhancers (eg, benzalkonium chloride) or iontophoresis leads to inadvertent partial or complete epi-off procedures. ${ }^{38-40}$ Other alternative approaches such as injection of riboflavin solution into an intrastromal pocket, ${ }^{41,42}$ topical application of riboflavin nanoemulsions ${ }^{43-45}$ or oral administration of riboflavin followed by sunlight exposure ${ }^{46}$ still await confirmation of effectiveness.

Clinical implementation of epi-on CXL techniques have shown variable efficacy. Limited comparisons of epion to epi-off techniques have not been sufficient to show differences in efficacy. ${ }^{47,48}$ However, epi-on techniques are compelling in that they avoid safety risks and slow visual recovery if they can be successfully implemented without disruption of the epithelium.

After sufficient riboflavin loading, oxygen is the limiting reagent in $\mathrm{CXL}^{49-51}$ However, with insufficient oxygen UVA radiation photodegrades riboflavin, reducing $\mathrm{CXL}_{\text {efficiency }}{ }^{52}$ and increasing UVA radiation exposure to corneal endothelium. In conventional CXL procedures, effective CXL and endothelial protection is achieved by repeated intraoperative application of riboflavin onto a deepithelialized stromal surface. This replaces riboflavin degraded by UVA exposure but can lead to marked variability in dosing and UVA transmission. ${ }^{53}$

The availability of oxygen determines the path of the interaction between riboflavin and UVA radiation. In lowoxygen conditions, the series of CXL reactions preferentially follow the Type 1 pathway, in which photoactivated triplet riboflavin reacts directly on substrates and is itself reduced, yielding hydrogen peroxide. In contrast, when sufficient oxygen is present, riboflavin transfers energy to dissolved oxygen to generate excited oxygen singlets, which are efficient cross-linkers (the Type 2 pathway). ${ }^{54}$ The Type 2 pathway preserves oxygen, preserves riboflavin, and does not produce toxic hydrogen peroxide.

We hypothesized that the addition of $\mathrm{NaI}$ to riboflavin would improve stromal riboflavin concentration during epi-on crosslinking. Pre-clinical studies of a riboflavin$\mathrm{NaI}$ solution showed consistent and adequate transepithelial penetration. ${ }^{55}$ A clinical study of epi-on crosslinking with the same formulation in 592 eyes showed promising results in the treatment of keratoconus and postoperative keratectasia; most eyes showed transepithelial saturation within 15 minutes and no repeat dosing was required. ${ }^{56}$ Sodium iodide is known to photo-stabilizes riboflavin in solution $^{57,58}$ and catalyzes conversion of hydrogen peroxide into oxygen and water. This study investigated the effect of $\mathrm{NaI}$ on riboflavin loading during topical application of a transepithelial riboflavin solution and on riboflavin degradation during subsequent UVA exposure in a rabbit model of epi-on CXL.

\section{Methods}

\section{Animals}

Twenty male New Zealand White rabbits (Charles River Laboratories, Wilmington, MA), weighing between 2.52 and 3.36 kilograms and $\sim 3.5-4.0$ months of age at study start, were housed under a 12/12 hour light/dark cycle with food and water provided ad libitum. All animals were healthy and free of clinically observable ocular disease.

Before assignment of animals to the study, each eye had a pre-screening examination consisting of indirect ophthalmoscopy and corneal slit-lamp examination (BP 
900, Haag-Streit AG, Bern, Switzerland) with photography to ensure that only normal corneas were used for experiments and to serve as a baseline for subsequent examinations.

All experimental protocols complied with the Association for Research in Vision and Ophthalmology Statement for the Use of Animals in Ophthalmic and Visual Research, the USDA Animal Welfare Act and the Public Health Service Policy on Humane Care and Use of Laboratory Animals. All animal activities were approved by an Institutional Animal Care and Use Committee (Absorption Systems California, LLC, San Diego, CA).

\section{Pilot Study}

A pilot study was performed to determine minimal time required for loading riboflavin prior to the main study. The procedure was the same as that detailed below with $3 \mathrm{mod}-$ ifications: riboflavin exposure was $12.5 \mathrm{~min}$, there was no UVA irradiation and both eyes of each animal were treated on the same day. Four rabbits were part of the pilot study; the remaining 16 were included in the main study.

\section{Riboflavin Solutions}

A formulation of $0.5 \%$ riboflavin 5 -phosphate and $0.015 \%$ $\mathrm{NaI}$ in half-normal saline (Ribostat, CXL Ophthalmics, Encinitas, CA) and an equivalent formulation without $\mathrm{NaI}$ were prepared by IriSys, LLC (San Diego, CA) and provided in ophthalmic droppers for application.

\section{Experimental Procedure}

Animals were anesthetized with intramuscular injections of ketamine $(50 \mathrm{mg} / \mathrm{kg})$ and xylazine $(10 \mathrm{mg} / \mathrm{kg})$. Each animal was treated using the transepithelial $\mathrm{NaI}$ riboflavin formulation in one eye and the NaI-free riboflavin formulation in the contralateral eye in a separate CXL procedure after a period of two days. Half the animals received the NaI riboflavin in the right eye and half in the left eye. Animals, eyes, and formulations were permuted according to a prospective randomization. One eye per animal was available for post-procedure bioanalysis.

All eyes were treated using the EpiSmart corneal crosslinking system consisting of NaI riboflavin (Ribostat, CXL Ophthalmics, Encinitas, CA) or the same riboflavin formulation with the NaI omitted, the EpiPrep wand, and the loading sponge applicators (CXL Ophthalmics, Encinitas, $\mathrm{CA}$ ). The wand was used to prepare the corneal surface. It is a proprietary, sterile device constructed of a rounded, nonabrasive, porous, hydrophilic sponge material bonded to a plastic handle. It is designed to cleanse the corneal surface of tear film mucins and surface lipids without disrupting the corneal epithelium. ${ }^{55,56}$ The wand was fully saturated with $0.5 \%$ proparacaine (Akorn, Inc, Lake Forest, IL) and the eye was gently brushed with minimal pressure in a circular motion over the entire cornea for $10-20 \mathrm{sec}$.

The riboflavin solutions were applied to the epithelial surface of the cornea using the EpiPrep loading sponge, a sterile, $12 \mathrm{~mm}$-diameter round device designed to conform to the cornea's curvature and provide homogeneous, continuous contact between the corneal epithelial surface and the riboflavin solution. ${ }^{53,54}$ The loading sponge was saturated with either the $\mathrm{NaI}$ or NaI-free transepithelial riboflavin solution and placed over the entire cornea for $12 \mathrm{~min}$, with short interruptions (24-49 sec, average 36 sec) for slit-lamp photographs and assessment of riboflavin loading as described below. To ensure continuous exposure to a full-concentration solution, 1-2 drops were added to the sponge approximately every $2 \mathrm{~min}$.

At the end of riboflavin loading, the eye was rinsed for approximately one second with balanced salt solution to remove residual surface riboflavin. Clearance was confirmed by slit-lamp examination. The eye was then irradiated with $4 \mathrm{~mW} / \mathrm{cm}^{2}$ of UVA radiation (CXLUSA Crosslinker, Theralight, LLC, Carlsbad, CA) with a 15 $\mathrm{sec}$ on $/ 15 \mathrm{sec}$ off cycle for $30 \mathrm{~min}$, with short interruptions (68-156 sec, average $105 \mathrm{sec})$ for slit-lamp photographs as described below. No further riboflavin solution was added during UV exposure, but balanced salt solution drops were applied periodically to prevent eye drying.

\section{Slit-Lamp Photography}

Slit-lamp photographs were taken at a standardized angle and illumination after the completion of riboflavin loading, immediately prior to the start of UVA exposure, and 10, $15,20,25$, and $30 \mathrm{~min}$ after the start of UVA exposure.

The slit-lamp images were assessed for riboflavin loading of the cornea. The intensity of riboflavin fluorescence in the corneal stroma was graded using a scale of 0-5, previously calibrated against liquid chromatography tandem mass spectroscopy (LC-MS/MS)-determined riboflavin concentration (Figure 1). ${ }^{56}$ Based on this and previous work, a threshold grade of 3 was determined to be sufficient for successful crosslinking. ${ }^{14,56}$

The examiner performing the grading was masked to the transepithelial riboflavin formulation administered. For the main study, the examiner was also masked to the time 


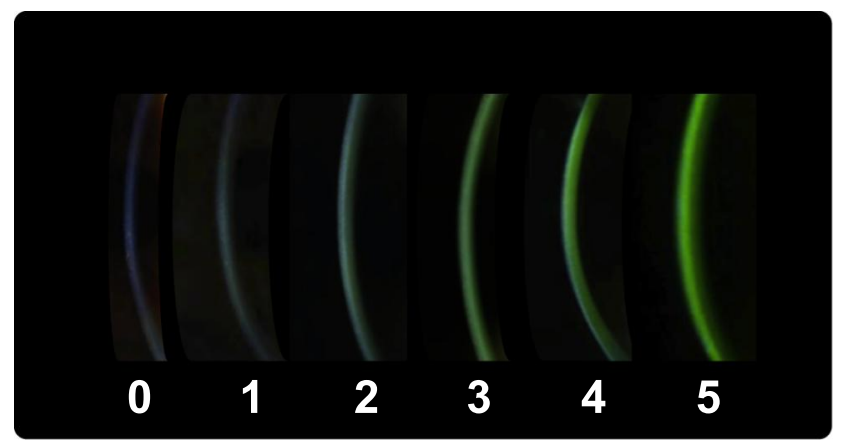

Figure I Stromal riboflavin grading scale. ${ }^{54}$ Grade 0: No green visible; Grade I: Mild green tint just visible; Grade 2: Substantial green visible; Grade 3: Obvious green color; Grade 4: Bright green appearance; Grade 5: Strong bright green color.

point at which the images were taken during UVA exposure.

Slit-lamp images were also used to perform clinical ophthalmic examinations to evaluate the eyes at each time point to assess ocular safety.

\section{Tissue Collection and Bioanalysis}

After completion of riboflavin loading, UVA exposure and slit-lamp imaging of the second eye, animals in the main study were humanely euthanized via pentobarbital overdose (150 mg/kg, intravenous). The euthanasia procedure was performed in compliance with the 2013 AVMA Guidelines for Euthanasia of Animals. Corneas were dissected from the eyes dosed on the day of euthanasia. The corneal epithelium and endothelium were debrided using $20 \%$ ethanol and the remaining corneal stroma collected and weighed. Corneal stromal samples from individual eyes were frozen at $-60^{\circ} \mathrm{C}$ to $-80^{\circ} \mathrm{C}$ until $\mathrm{LC}-\mathrm{MS} / \mathrm{MS}$ analysis to determine the concentrations of riboflavin and riboflavin 5'-phosphate (methods are detailed in reference 55).

\section{Data Analysis}

Riboflavin grades for eyes treated with the transepithelial riboflavin formulation with or without NaI were compared using a two-way mixed-design ANOVA with Time as the within-subjects factor and Riboflavin Formulation as the between-subjects factor. Where appropriate, post hoc testing was performed using the Sidak test. To evaluate the differential rates of riboflavin degradation during UVA exposure, differences in riboflavin grades between time $=$ 0 (the beginning of UVA exposure) and subsequent time points were calculated for each sample. The change-frombaseline in these grades was compared using the same analysis. Riboflavin and riboflavin 5'-phosphate concentrations in the corneal stroma after treatment with transepithelial riboflavin formulation with or without $\mathrm{NaI}$ were compared using unpaired two-tailed t-tests. The level of significance was set at 0.05 . Data were analyzed using GraphPad Prism software, Version 6.07 (GraphPad Software, San Diego, CA).

\section{Results}

At baseline, all ophthalmic observations were normal for all enrolled animals.

\section{Pilot Study}

Riboflavin fluorescence in the corneal stroma increased with increasing riboflavin loading time (Table S1). Mean riboflavin grades for both transepithelial riboflavin formulations were consistent with the target grade for crosslinking at the $12.5 \mathrm{~min}$ observation after the start of the loading procedure (Figure 2). At this time point, all eyes had reached a riboflavin grade of 3 regardless of formulation. Based on this finding, 12 min was selected as the riboflavin loading time for the main study.

\section{Main Study}

Immediately after the riboflavin loading procedure, eyes treated with either $\mathrm{NaI}$ riboflavin or $\mathrm{NaI}$-free riboflavin formulation exhibited riboflavin loading (Table S2). Mean riboflavin grades were higher in eyes treated with the NaI-containing formulation $(2.94 \pm 0.57)$ compared to eyes treated with the formulation without $\mathrm{NaI}(2.56 \pm$ 0.63 ) (Figure 3). Overall, of the 96 data points collected

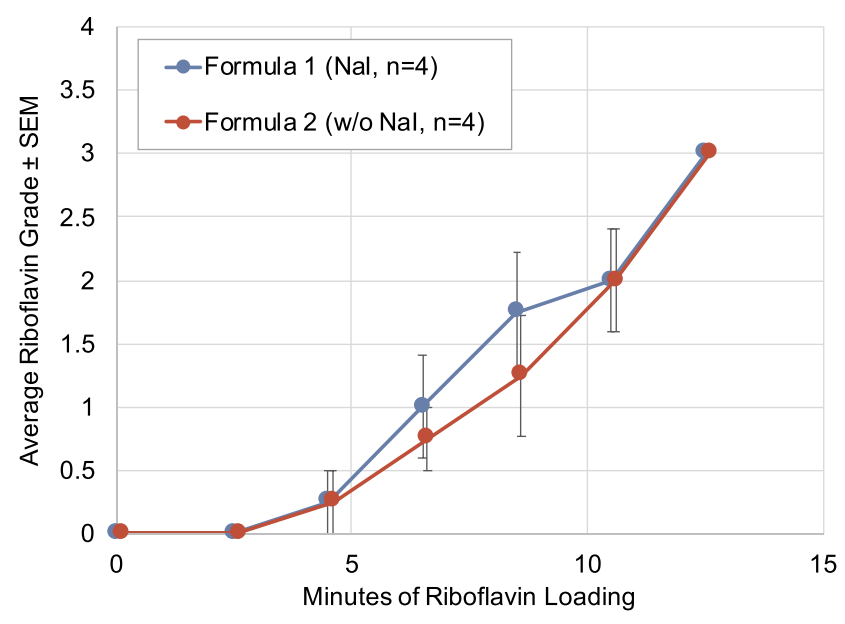

Figure 2 Riboflavin grade vs riboflavin loading time in eyes treated with transepithelial riboflavin formulation with or without $\mathrm{Nal}$ in the pilot study $(n=4$ eyes per formulation).

Abbreviation: SEM, standard error of the mean. 
for each eye-timepoint per formulation $63(66 \%)$ were at or above the fluorescence grade of 3 for the NaIcontaining formulation, while $33(34 \%)$ were at or above the fluorescence grade of 3 for the non-NaI formulation.

Mean riboflavin grades in both treatment groups increased slightly over the first $10 \mathrm{~min}$ of UVA exposure and then gradually decreased over the course of the remaining UVA exposure. Riboflavin grades in eyes treated with the $\mathrm{NaI}$ riboflavin remained higher than those in eyes treated with the NaI-free formulation throughout UVA exposure (Figure 3). The ANOVA detected highly significant main effects $(P<0.001)$ of both Riboflavin Formulation and Time. No interaction between the two factors was observed. Post hoc testing found statistically significant differences $(P<$ 0.05 ) between the treatment groups at the 15, 20, and $25 \mathrm{~min}$ time points. The same significance levels (Figure 3) were found with Bonferroni-adjusted Wilcoxon ranked-sum tests.

Mean riboflavin grades decreased more quickly over the initial course of UVA exposure in eyes treated with the $\mathrm{NaI}$-free formulation compared to eyes treated with the $\mathrm{NaI}$ riboflavin. This was reflected in a steady increase in the difference in mean riboflavin grade between the two treatment groups, peaking at 25 min of UVA exposure. The ANOVA of the baseline-subtracted grades throughout UVA exposure showed a trend towards less degradation over time with the NaI-containing formulation, but the difference did not reach significance $(P=0.12)$

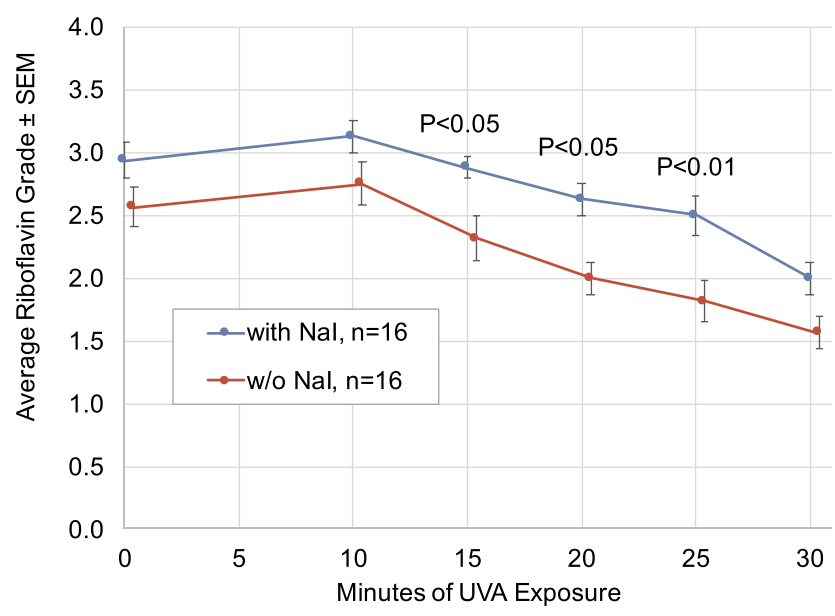

Figure 3 Riboflavin grade vs time throughout UVA exposure in eyes treated with transepithelial riboflavin formulation with or without $\mathrm{Nal}(n=16$ eyes per formulation). Mean riboflavin grade immediately after the riboflavin loading procedure was higher in eyes treated with $\mathrm{Nal}$ compared to eyes treated with $\mathrm{Nal}$-free riboflavin formulation. Mean riboflavin grade remained higher in eyes treated with $\mathrm{Nal}$ compared to eyes treated with $\mathrm{Nal}$-free riboflavin formulation throughout UVA exposure. Two-way mixed-design ANOVA analysis showed highly significant $(p<0.00 \mathrm{I})$ differences in overall grade throughout UVA exposure and for change in grade over time. Individual time point $P$-values are from Sidak post hoc test after ANOVA.
Mean riboflavin concentration in the corneal stroma after the procedure and sacrifice was comparable in eyes treated with either formulation ( $\mathrm{NaI}$ riboflavin: $49.7 \pm 11.3$ $\mu \mathrm{g} / \mathrm{g}$; NaI-free riboflavin: $48.3 \pm 8.2 \mu \mathrm{g} / \mathrm{g}$ ) (Figure 4A and Table S3). However, mean riboflavin 5'-phosphate concentration was higher in eyes treated with $\mathrm{NaI}$ riboflavin $(10.3$ $\pm 3.7 \mu \mathrm{g} / \mathrm{g}$ ) compared to those treated with the NaI-free formulation $(7.7 \pm 2.0 \mu \mathrm{g} / \mathrm{g})$ (Figure 4B and Table S4). While the difference did not reach statistical significance, a trend was observed $(\mathrm{P}=0.095)$.

Clinical examination findings were limited to mild conjunctival congestion at most time points, with a few instances of transient moderate congestion; mild opacity of the corneal epithelium immediately after riboflavin loading that resolved in most eyes within $10 \mathrm{~min}$ and a single case of conjunctival swelling starting $15 \mathrm{~min}$ into UVA exposure. Findings occurred with comparable frequency and severity in eyes treated with the transepithelial riboflavin formulation with or without NaI (Table 1). No evidence of epithelial disruption was seen after corneal loading and throughout UVA exposure, with the exception of a single instance of a small debrided area of the corneal epithelium in one eye treated with the NaI-free riboflavin formulation. No non-ocular health effects were observed.

\section{Discussion}

Topical application of an optimized riboflavin formulation with $\mathrm{NaI}$ or an otherwise identical riboflavin formulation without NaI using the EpiSmart system resulted in robust transepithelial riboflavin delivery into corneal stroma. Higher riboflavin slit-lamp grades were observed immediately after riboflavin application when $\mathrm{NaI}$ was present in the formulation, indicating greater stromal riboflavin concentration. Highly significant main effects were found for both Riboflavin Formulation and Time, indicating a significant difference between eyes treated with the different riboflavin formulations throughout the time course of UVA exposure, but also a decrease in riboflavin concentration throughout the UV exposure with both formulations. The significant difference in grade at the 15,20 , and 25 minute-timepoints may be the result of $\mathrm{NaI}$ improving riboflavin delivery into the stroma across the intact corneal epithelium and/or providing some photoprotection of riboflavin during UVA exposure.

The pilot study showed sufficient riboflavin (grade $=3$ ) after 12.5 minutes of loading in all 8 eyes of 4 rabbits. During the main study, a consistent loading time was used, but some variability was seen among the 32 eyes tested 
A

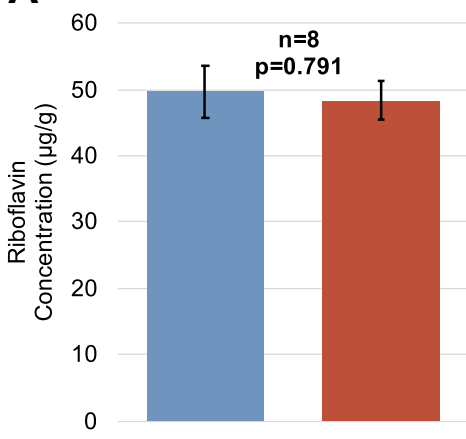

B

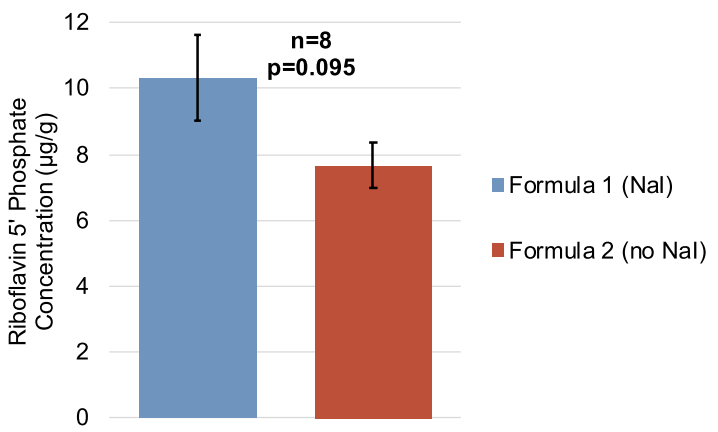

Figure 4 Concentrations of riboflavin and riboflavin 5 '-phosphate in the corneal stroma in eyes ( $\mathrm{n}=8$ per formulation) treated with transepithelial riboflavin formulation with or without Nal after UVA exposure. (A) Mean riboflavin concentrations. (B) Mean riboflavin 5'-phosphate concentrations; $P$-values are from unpaired two-tailed $t$-test. Data shown as mean \pm standard error of the mean.

(16 eyes in each cohort), with the NaI-containing formula cohort achieving a higher mean level of stromal riboflavin than the NaI-free formulation. It is possible that the NaIriboflavin formula resulted, on average, in improved transepithelial delivery of riboflavin. However, the study was planned and powered to detect differences of $\geq 0.5$ gradelevels after UV exposure, and the 0.38 difference at time $=$ 0 did not reach statistical significance.

Both formulations demonstrated an increase in mean riboflavin grade by the 10 minute time point during UVA exposure relative to the time of initiating exposure. In a previous study of $\mathrm{NaI}$ riboflavin, ${ }^{55}$ riboflavin grades increased for 15-20 min after completion of NaI riboflavin application, ${ }^{55}$ in the absence of UVA exposure; presumably due to further permeation and homogenization. In this prior study, the $\mathrm{NaI}$ riboflavin grade rose to $4+$ on average,${ }^{55}$ while the peak grade in the current study was approximately 3 . The relatively lower grades in this study may be due to a lower initial concentration of riboflavin or to photodegradation within the first 10 minutes of UVA exposure. Throughout the UVA exposure, $66 \%$ of the NaI-riboflavin treated eyes maintained the target grade of 3 , while $33 \%$ of the non-NaI treated eyes did so, indicating that an eye treated with $\mathrm{NaI}$ was roughly twice as likely to have a concentration of riboflavin sufficient for crosslinking at any given time during the CXL procedure.

Riboflavin grades decreased during UVA exposure of the cornea, starting $10 \mathrm{~min}$ after completion of riboflavin

Table I Clinical Examination Findings in Eyes Treated with Transepithelial Riboflavin Formulation with or without Nal

\begin{tabular}{|c|c|c|c|c|c|c|c|}
\hline \multirow[t]{2}{*}{ Finding } & \multirow[t]{2}{*}{$\begin{array}{l}\text { Treatment } \\
\text { Group }\end{array}$} & $\begin{array}{c}0 \\
\min \end{array}$ & $\begin{array}{c}10 \\
\min \end{array}$ & $\begin{array}{l}15 \\
\min \end{array}$ & $\begin{array}{c}20 \\
\min \end{array}$ & $\begin{array}{c}25 \\
\min \end{array}$ & $\begin{array}{c}30 \\
\min \end{array}$ \\
\hline & & \multicolumn{6}{|c|}{$\begin{array}{c}\text { Number of Eyes Affected (of } \mathrm{N}=16 \text { Total per } \\
\text { Treatment Group) }\end{array}$} \\
\hline \multirow[t]{2}{*}{ Mild conjunctival congestion } & With Nal & 14 & 13 & 15 & 15 & 16 & 16 \\
\hline & Without Nal & 16 & 13 & 14 & 14 & 15 & 15 \\
\hline \multirow[t]{2}{*}{ Moderate conjunctival congestion } & With Nal & 1 & 2 & 1 & 1 & 0 & 0 \\
\hline & Without Nal & 0 & 3 & 2 & 2 & I & I \\
\hline \multirow[t]{2}{*}{ Mild conjunctival swelling } & With Nal & 0 & 0 & 0 & 0 & 0 & 0 \\
\hline & Without Nal & 0 & 0 & 1 & 1 & 1 & 1 \\
\hline \multirow{2}{*}{$\begin{array}{l}\text { Mild corneal opacity affecting } I-25 \% \text { of corneal } \\
\text { area }\end{array}$} & With Nal & 6 & 2 & 1 & 1 & 1 & I \\
\hline & Without Nal & 3 & 4 & 3 & 3 & I & I \\
\hline \multirow[t]{2}{*}{ Mild corneal opacity affecting $>\mathbf{2 5} \%$ of corneal area } & With Nal & 10 & 0 & 0 & 0 & 0 & 0 \\
\hline & Without Nal & 13 & 0 & 0 & 0 & 0 & 0 \\
\hline
\end{tabular}


application and onset of UVA exposure. This pattern reflects photodegradation of riboflavin in the corneal stroma by UVA radiation. In eyes treated with the $\mathrm{NaI}$ riboflavin formulation, riboflavin grades decreased more slowly during UVA exposure compared to eyes treated with the NaI-free formulation. This finding suggests that $\mathrm{NaI}$ had a photoprotective effect, slowing the photodegradation of riboflavin by UVA. In shelf stability studies, $\mathrm{NaI}$ effectively blocks the photodegradation of riboflavin compared to the same riboflavin formulation without NaI (unpublished data on file, IriSys, San Diego, CA). This is consistent with the known action of iodide to quench photo-excited states of riboflavin which, in anaerobic conditions, cause it to self-reduce and degrade. ${ }^{54,57,58}$

We have hypothesized that Iodide ions would also slow anaerobic photoreactions with riboflavin in vivo, resulting in reduced photodegradation and more efficient aerobic crosslinking. Trace amounts of iodide ions quench the long-lived photo-excited triplet riboflavin to its ground state before it can interact with substrates and be degraded in Type 1 reactions. ${ }^{57,58}$ It is expected that iodide will slow crosslinking reactions when oxygen is limited while allowing it to proceed when oxygen is sufficient to compete successfully to accept energy from riboflavin in the excited triplet state, yielding ground-state riboflavin and excited oxygen singlets. Oxygen singlets would then cross-link protein substrates via Type 2 interactions. A smaller number of Type 1 reactions will occur, yielding less hydrogen peroxide, which is long-lived and cytotoxic. However, the iodide ion's well-known catalytic action on peroxide would quickly restore diatomic oxygen to the solution for further crosslinking reactions (Figure 5). The increased riboflavin concentration seen in the present study is consistent with this shift from the Type 1 pathway to the favorable Type 2 CXL photoreaction pathway.

If riboflavin degradation is inhibited in the corneal stroma during a clinically relevant time window, ie, over 20-30 min of UVA exposure, ${ }^{51}$ then NaI may be helpful to in vivo crosslinking. The data in the present study do not reach statistical significance in showing this independent effect in terms of a decay slope, but do show significantly higher riboflavin grades at 15,20 , and 25 minutes after onset of UVA exposure during crosslinking. Observations of iodide's protective effect have shown that in later stages of photoreaction, Type 1 reactions accumulate and reduce riboflavin concentration. ${ }^{57}$ Similarly, the data here show some decrease in the relative benefit of the NaI-containing formula after 25 minutes of UVA exposure.

Bioanalysis of corneal stroma after riboflavin loading and UVA exposure confirmed a trend toward higher stromal levels of riboflavin $5^{\prime}$-phosphate in eyes treated with the NaI-containing riboflavin solution, but without statistical significance. Notably, this difference was detected after the completion of the full 30-minute course of UVA exposure, when the difference between treatment groups had started to diminish. Bioanalysis of corneas collected at the point of greatest difference in riboflavin grade between treatment groups (at $\leq 25$ min of UVA exposure) may more readily reveal a statistically significant difference in stromal concentrations.

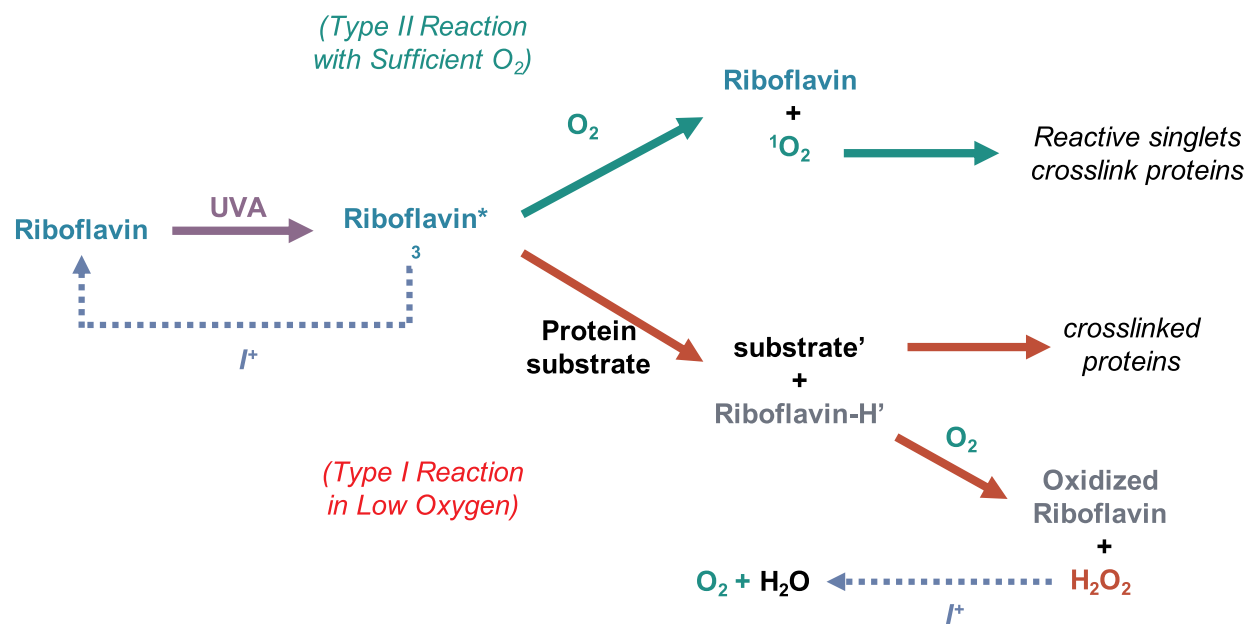

Figure 5 Illustration of the two possible reaction pathways for the crosslinking (CXL) photoreaction and hypothesized action of iodide. In low-oxygen conditions, the reaction follows the Type I pathway (red arrows), in which excited riboflavin triplets (Riboflavin* ${ }_{3}$ ), acting as a cross-linker, reacts with substrate forming radicals (substrate' and Riboflavin- $\mathrm{H}^{\prime}$ ), and then with water to form hydrogen peroxide and derivatives of riboflavin. In the presence of sufficient oxygen, riboflavin reacts directly with oxygen to generate less toxic singlet oxygen $\left({ }^{\prime} \mathrm{O}_{2}\right)$ that cross-links extracellular matrix proteins (Type 2 pathway, green arrows). Sodium iodide (blue dotted arrows) shifts the $\mathrm{CXL}$ photoreaction from the toxic Type I pathway to favorable Type 2 pathway by quenching excited triplet riboflavin in anaerobic conditions. Further, if Type I reactions occur, iodide promotes the immediate conversion of hydrogen peroxide into oxygen and water, providing diatomic oxygen for further crosslinking reactions. 
The procedure was well tolerated in all eyes. Clinical findings in the study were limited to transient, minor, conjunctival irritation, mostly evidenced by mild to moderate conjunctival congestion. Mild corneal epithelium opacity seen after riboflavin loading resolved over the course of UVA exposure. Clinical findings were comparable in eyes treated with either the $\mathrm{NaI}$ riboflavin or the $\mathrm{NaI}$-free riboflavin formulation, indicating that the $\mathrm{NaI}$ was well tolerated.

This study is limited by the lack of intermediate quantitation of riboflavin in stromal tissue during UVA exposure, as was done with LC-MS/MS after the 30 minute timepoint. The semi-quantitative rating of masked slit-lamp riboflavin fluorescence correlates well with results from LC-MS/MS analysis, ${ }^{55}$ but may have lacked the precision to discriminate between potential penetration effects and photoprotective effects of the $\mathrm{NaI}$ additive. The inability to analyze both eyes per animal post-mortem also contributed to uncertainty, because only 8 of 16 eyes per group were available for analysis. Further, a fixed loading time was used, which may have provided a suboptimal riboflavin concentration. A 20minute loading time was typically sufficient in clinical studies, ${ }^{56}$ but the optimal time in rabbits is not wellvalidated. The penetration effect warrants further careful study. Finally, the specific effects of iodide may be more or less apparent using different protocols. It is possible that the interruptions of the UVA exposure may have allowed for oxygen rediffusion which blunted the differential photoprotective effect of iodide we sought to observe. The riboflavin loading sponge devices and protocol, as well as the 15-second cycling time for UVA exposure was optimized in the clinic with the same objective of the iodide additive: to maintain oxygen and a high concentration of riboflavin throughout the crosslinking procedure.

These findings demonstrate that the addition of $\mathrm{NaI}$ to the transepithelial riboflavin formulation slows the reduction of riboflavin concentration throughout a clinically relevant course of UVA exposure. This may be accomplished by a combination of enhancing penetration of riboflavin across the intact epithelium into the corneal stroma and photo-protecting riboflavin, limiting riboflavin photodegradation during UVA exposure.

\section{Acknowledgments}

We would like to thank Nurith Amitai-Crawford, $\mathrm{PhD}$ for her assistance preparing this manuscript.

\section{Funding}

Study funded by CXL Ophthalmics, LLC, contributions by Absorption Systems and CXLUSA, LLC.

\section{Disclosure}

Roy S Rubinfeld equity interests in CXL Ophthalmics, LLC, Encinitas, CA, CurveRight, LLC, Bethesda, MD and CXLUSA, LLC, Bethesda, MD, and has a patent 15/335,146 issued, a patent 13768403.1 , a patent $14 / 387,803$, a patent 14 / 387,532 , a patent $14 / 275,192$, a patent 13767439.6 , a patent 15793441.5 , a patent $14 / 206,847$, a patent $15 / 271,668$, and a patent 14775693.6; Jonathan $\mathrm{H}$ Talamo has equity interest in and serves on the Board of Directors for CXL Ophthalmics, LLC; Edward C Parsons is a consultant for CXL Ophthalmics, LLC, Encinitas, CA; Glenwood G Gum has no conflicts of interest to disclose.

\section{References}

1. Wollensak G, Spoerl E, Seiler T. Riboflavin/ultraviolet-A-induced collagen crosslinking for the treatment of keratoconus. $\mathrm{Am}$ $J$ Ophthalmol. 2003;135(5):620-627. doi:10.1016/S0002-9394(02) 02220-1

2. Zhang Y, Conrad AH, Conrad GW, et al. Effects of ultraviolet-A and riboflavin on the interaction of collagen and proteoglycans during corneal cross-linking. J Biol Chem. 2011;286(15):13011-13022. doi:10.1074/jbc.M110.169813

3. Spoerl E, Huhle M, Seiler T. Induction of cross-links in corneal tissue. Exp Eye Res. 1998;66(1):97-103. doi:10.1006/exer.1997.0410

4. Hersh PS, Greenstein SA, Fry KL. Corneal collagen crosslinking for keratoconus and corneal ectasia: one-year results. $J$ Cataract Refract Surg. 2011;37(1):149-160. doi:10.1016/j.jcrs.2010.07.030

5. O'Brart DPS, Kwong TQ, Patel P, et al. Long-term follow-up of riboflavin/ultraviolet A $(370 \mathrm{~nm})$ corneal collagen cross-linking to halt the progression of keratoconus. Br J Ophthalmol. 2013;97 (4):433-437. doi:10.1136/bjophthalmol-2012-302556

6. Raiskup F, Theuring A, Pillunat LE, et al. Corneal collagen crosslinking with riboflavin and ultraviolet-A light in progressive keratoconus: ten-year results. J Cataract Refract Surg. 2015;41(1):41-46. doi:10.1016/j.jcrs.2014.09.033

7. Wittig-Silva C, Chan E, Islam FMA, et al. A randomized, controlled trial of corneal collagen cross-linking in progressive keratoconus. Ophthalmology. 2014;121(4):812-821. doi:10.1016/j.ophtha.201 3.10 .028

8. Dhawan S, Rao K, Natrajan S. Complications of corneal collagen cross-linking. $J$ Ophthalmol. 2011;2011:1-5. doi:10.1155/2011/ 869015

9. Goldich Y, Marcovich AL, Barkana Y, et al. Safety of corneal collagen cross-linking with UV-A and riboflavin in progressive keratoconus. Cornea. 2010;29(4):409-411. doi:10.1097/ICO.0b01 3e3181bd9f8c

10. Greenstein SA, Fry KL, Bhatt J, et al. Natural history of corneal haze after collagen crosslinking for keratoconus and corneal ectasia: scheimpflug and biomicroscopic analysis. J Cataract Refract Surg. 2010;36(12):2105-2114. doi:10.1016/j.jcrs.2010.06.067

11. Raiskup F, Hoyer A, Spoerl E. Permanent corneal haze after riboflavin-UVA-induced cross-linking in keratoconus. $J$ Refract Surg. 2009;25(9):S824-S828. doi:10.3928/1081597X-20090813-12 
12. $\mathrm{Xu} \mathrm{K}$, Chan TCY, Vajpayee RB, et al. Corneal collagen cross-linking. Asia Pac J Ophthalmol (Phila). 2015;4(5):300-306. doi:10.1097/APO.0000000000000145

13. Hammer A, Rudaz S, Guinchard S, et al. Analysis of riboflavin compounds in the rabbit cornea in vivo. Curr Eye Res. 2016;41 (9):1166-1172. doi:10.3109/02713683.2015.1101141

14. Baiocchi S, Mazzotta C, Cerretani D, et al. Corneal crosslinking: riboflavin concentration in corneal stroma exposed with and without epithelium. J Cataract Refract Surg. 2009;35(5):893-899. doi:10.1016/j.jcrs.2009.01.009

15. Gore DM, O'Brart D, French P, et al. Transepithelial riboflavin absorption in an ex vivo rabbit corneal model. Invest Ophthalmol Vis Sci. 2015;56(8):5006-5011. doi:10.1167/iovs.15-16903

16. Samaras K, O'Brart DP, Doutch J, et al. Effect of epithelial retention and removal on riboflavin absorption in porcine corneas. $J$ Refract Surg. 2009;25(9):771-775. doi:10.3928/1081597X-20090813-03

17. Kissner A, Spoerl E, Jung R, et al. Pharmacological modification of the epithelial permeability by benzalkonium chloride in UVA/riboflavin corneal collagen cross-linking. Curr Eye Res. 2010;35 (8):715-721.doi:10.3109/02713683.2010.481068

18. Leccisotti A, Islam T. Transepithelial corneal collagen cross-linking in keratoconus. J Refract Surg. 2010;26(12):942-948. doi:10.3928/ 1081597X-20100212-09

19. Caporossi A, Mazzotta C, Paradiso AL, et al. Transepithelial corneal collagen crosslinking for progressive keratoconus: 24-month clinical results. J Cataract Refract Surg. 2013;39(8):1157-1163. doi:10.1016/ j.jcrs.2013.03.026

20. Wollensak G, Iomdina E. Biomechanical and histological changes after corneal crosslinking with and without epithelial debridement. $J$ Cataract Refract Surg. 2009;35(3):540-546. doi:10.1016/j. jcrs.2008.11.036

21. Armstrong BK, Lin MP, Ford MR, et al. Biological and biomechanical responses to traditional epithelium-off and transepithelial riboflavin-UVA CXL techniques in rabbits. J Refract Surg. 2013;29 (5):332-341. doi:10.3928/1081597X-20130415-04

22. Morrison PWJ, Connon CJ, Khutoryanskiy VV. Cyclodextrinmediated enhancement of riboflavin solubility and corneal permeability. Mol Pharm. 2013;10(2):756-762. doi:10.1021/ mp3005963

23. Ostacolo C, Caruso C, Tronino D, et al. Enhancement of corneal permeation of riboflavin-5'-phosphate through vitamin E TPGS: a promising approach in corneal trans-epithelial cross linking treatment. Int $J$ Pharm. 2013;440(2):148-153. doi:10.1016/j. ijpharm.2012.09.051

24. Caruso C, Ostacolo C, Epstein RL, et al. Transepithelial corneal cross-linking with vitamin E-enhanced riboflavin solution and abbreviated, low-dose UV-A. Cornea. 2016;35(2):145-150. doi:10.1097/ ICO.0000000000000699

25. Rechichi M, Daya S, Scorcia V, et al. Epithelial-disruption collagen crosslinking for keratoconus: one-year results. J Cataract Refract Surg. 2013;39(8):1171-1178. doi:10.1016/j.jcrs.2013.05.022

26. Kaya V, Utine CA, Yilmaz OF. Efficacy of corneal collagen cross-linking using acustom epithelial debridement technique in thin corneas: a confocal microscopy study. J Refract Surg. 2010;27 (6):444-450. doi:10.3928/1081597X-20101201-01

27. Hashemi H, Miraftab M, Hafezi F, et al. Matched comparison study of total and partial epithelium removal in corneal cross-linking. $J$ Refract Surg. 2015;31(2):110-115. doi:10.3928/1081597X20150122-06

28. Gore DM, O'Brart DP, French P, et al. A comparison of different corneal iontophoresis protocols for promoting transepithelial riboflavin penetration. Invest Ophthalmol Vis Sci. 2015;56(13):7908-7914. doi:10.1167/iovs.15-17569

29. Lamy R, Chan E, Zhang H, et al. Ultrasound-enhanced penetration of topical riboflavin into the corneal stroma. Invest Ophthalmol Vis Sci. 2013;54(8):5908-5912. doi:10.1167/iovs.13-12133
30. Franch A, Birattari F, Dal Mas G, et al. Evaluation of intrastromal riboflavin concentration in human corneas after three corneal cross-linking imbibition procedures: a pilot study. J Ophthalmol. 2015;2015:794256. doi:10.1155/2015/794256

31. Buzzonetti L, Petrocelli G, Valente P, et al. Iontophoretic transepithelial corneal cross-linking to halt keratoconus in pediatric cases: 15-month follow-up. Cornea. 2015;34(5):512-515. doi:10.1097/ ICO.0000000000000410

32. Li N, Fan Z, Peng X, et al. Clinical observation of transepithelial corneal collagen cross-linking by iontophoresis of riboflavin in treatment of keratoconus. Eye Sci. 2014;29(3):160-164. PMID: 2611972

33. Vinciguerra P, Randleman JB, Romano V, et al. Transepithelial iontophoresis corneal collagen cross-linking for progressive keratoconus: initial clinical outcomes. J Refract Surg. 2014;30(11):746-753. doi:10.3928/1081597X-20141021-06

34. Soeters N, Wisse RPL, Godefrooij DA, et al. Transepithelial versus epithelium-off corneal cross-linking for the treatment of progressive keratoconus: a randomized controlled trial. Am J Ophthalmol. 2015;159(5):821-828.e3. doi:10.1016/j.ajo.2015.02.005

35. Jia H, Peng X. Efficacy of iontophoresis-assisted epithelium-on corneal cross-linking for keratoconus. Int $J$ Ophthalmol. 2018;11 (4):687-694. doi:10.18240/ijo.2018.04.25

36. Al Fayez MF, Alfayez S, Alfayez Y. Transepithelial versus epithelium-off corneal collagen cross-linking for progressive keratoconus: a prospective randomized controlled trial. Cornea. 2015;34 (Suppl 10):S53-56. doi:10.1097/ICO.0000000000000547

37. Hirji N, Sykakis E, Lam FC, et al. Corneal collagen crosslinking for keratoconus or corneal ectasia without epithelial debridement. Eye. 2015;29(6):764-768. doi:10.1038/eye.2015.23

38. Gatzioufas Z, Raiskup F, O'Brart D, et al. Transepithelial corneal cross-linking using an enhanced riboflavin solution. J Refract Surg 2016;32(6):372-377. doi:10.3928/1081597X-20160428-02

39. Yuksel E, Novruzlu S, Ozmen MC, et al. A study comparing standard and transepithelial collagen cross-linking riboflavin solutions: epithelial findings and pain scores. J Ocul Pharmacol Ther. 2015;31 (5):296-302. doi:10.1089/jop.2014.0090

40. Taneri S, Oehler S, Lytle G, et al. Evaluation of epithelial integrity with various transepithelial corneal cross-linking protocols for treatment of keratoconus. J Ophthalmol. 2014;2014:1-5. doi:10.1155/ 2014/614380

41. Wollensak G, Hammer CM, Spörl E, et al. Biomechanical efficacy of collagen crosslinking in porcine cornea using a femtosecond laser pocket. Cornea. 2014;33(3):300-305. doi:10.1097/ICO.0000000 000000059

42. Kanellopoulos AJ. Collagen cross-linking in early keratoconus with riboflavin in a femtosecond laser-created pocket: initial clinical results. J Refract Surg. 2009;25(11):1034-1037. doi:10.3928/ 1081597X-20090901-02

43. Labate C, Lombardo M, Lombardo G, et al. Biomechanical strengthening of the human cornea induced by nanoplatform-based transepithelial riboflavin/UV-A corneal cross-linking. Invest Opthalmol Vis Sci. 2017;58(1):179. doi:10.1167/iovs.16-20813

44. Bottos KM, Oliveira AG, Bersanetti PA, et al. Corneal absorption of a new riboflavin-nanostructured system for transepithelial collagen cross-linking. PLoS One. 2013;8(6):e66408. doi:10.1371/journal. pone.0066408

45. Lombardo G, Micali NL, Villari V, et al. Assessment of stromal riboflavin concentration-depth profile in nanotechnology-based transepithelial corneal crosslinking. J Cataract Refract Surg. 2017;43 (5):680-686. doi:10.1016/j.jcrs.2017.03.026

46. University of Missouri-Columbia. Dietary riboflavin (Vitamin B-2) and cornea cross-linking. Available from: https:/clinicaltrials.gov/ ct2/show/NCT03095235. NLM identifier: NCT03095235. Accessed August 24, 2020.. 
47. Zhang X, Zhao J, Li M, et al. Conventional and transepithelial corneal cross-linking for patients with keratoconus. PLoS One. 2018;13(4):e0195105. doi:10.1371/journal.pone.0195105

48. Niyazmand H, McKelvie J, Li Y, McLintock C. Comparison of visual and tomographic outcomes of epithelium-on and epithelium-off accelerated corneal crosslinking: a longitudinal Study. Cornea. 2020. doi:10.1097/ICO.0000000000002567

49. Ng ALK, Kwok PSK, Wu RTK, et al. Comparison of the demarcation line on ASOCT after simultaneous lasik and different protocols of accelerated collagen crosslinking. Cornea. 2017;36(1):74-77. doi:10.1097/ICO.0000000000001012

50. Richoz O, Hammer A, Tabibian D, et al. The biomechanical effect of corneal collagen cross-linking (CXL) with riboflavin and UV-A is oxygen dependent. Transl Vis Sci Technol. 2013;2(7):6. doi:10.1167/ tvst.2.7.6

51. Rubinfeld RS, Caruso C, Ostacolo C. Corneal cross-linking: the science beyond the myths and misconceptions. Cornea. 2019;38 (6):780-790. doi:10.1097/ICO.0000000000001912

52. Caruso C, Barbaro G, Epstein RL, et al. Corneal cross-linking. Cornea. 2016;35(5):659-662. doi:10.1097/ICO.0000000000000809
53. Caruso C, Epstein RL, Ostacolo C, et al. Customized corneal crosslinking-A mathematical model. Cornea. 2017;36(5):600-604. doi:10.1097/ICO.0000000000001160

54. Kamaev P, Friedman MD, Sherr E, et al. Photochemical kinetics of corneal cross-linking with riboflavin. Invest Opthalmol Vis Sci. 2012;53(4):2360. doi:10.1167/iovs.11-9385

55. Rubinfeld RS, Stulting RD, Gum GG, et al. Quantitative analysis of corneal stromal riboflavin concentration without epithelial removal. $J$ Cataract Refract Surg. 2018;44(2):237-242. doi:10.1016/j. jcrs.2018.01.010

56. Stulting RD, Trattler WB, Woolfson JM, et al. Corneal crossinking without epithelial removal. J Cataract Refract Surg. 2018;44 (11):1363-1370. doi:10.1016/j.jcrs.2018.07.029

57. Holmström B, Oster G. Riboflavin as an electron donor in photochemical reactions. J Am Chem Soc. 1961;83(8):1867-1871. doi:10.1021/ja01469a022

58. Sheraz MA, Kazi SH, Ahmed S, Anwar Z, Ahmad I. Photo, thermal and chemical degradation of riboflavin. Beilstein J Org Chem. 2014;10:1999-2012. doi:10.3762/bjoc.10.208
Clinical Ophthalmology

\section{Publish your work in this journal}

Clinical Ophthalmology is an international, peer-reviewed journal covering all subspecialties within ophthalmology. Key topics include: Optometry; Visual science; Pharmacology and drug therapy in eye diseases; Basic Sciences; Primary and Secondary eye care; Patient Safety and Quality of Care Improvements. This journal is indexed on PubMed

\section{Dovepress}

Central and CAS, and is the official journal of The Society of Clinical Ophthalmology (SCO). The manuscript management system is completely online and includes a very quick and fair peer-review system, which is all easy to use. Visit http://www.dovepress.com/ testimonials.php to read real quotes from published authors. 